\title{
PLAN DE NEGOCIOS PARA LA EXPORTACIÓN DE EMPANIZADOS DE CALAMAR GIGANTE AL MERCADO DE ESTADOS UNIDOS*
}

\author{
Liliana Baca \\ lili_baca@hotmail.com \\ Andreína González \\ andreinagsapene@hotmail.com \\ José Méndez \\ jmendez_79@hotmail.com \\ Tatiana Urrunaga \\ tatiu@hotmail.com
}

\section{Resumen}

Este artículo desarrolla un plan de negocios para introducir el calamar gigante en el mercado de Estados Unidos en la forma de empanizados marinos. El valor agregado que el procesamiento local le otorga al recurso es determinante para obtener precios muy superiores en comparación con el que se paga por el calamar como materia prima, y para ingresar en los mercados internacionales de productos terminados. El modelo planteado comprende el plan de márketing, la estrategia de operaciones y la evaluación económica; esta última muestra los resultados económicos esperados y la rentabilidad generada. El artículo termina señalando los factores críticos del plan de negocios: las características de la demanda en el mercado de destino y el aprovisionamiento de la materia prima.

Palabras clave: calamar, empanizados marinos, exportación, Estados Unidos.

\begin{abstract}
This article develops a business plan to introduce giant squid in markets in the United States in the form of breaded seafood. The added value that local processing gives to the resource is determinant for obtaining higher prices, as compared to those being paid for squid as raw material, and for entering international markets for finished products. The proposed model covers the marketing plan, operations strategies and economic evaluation; the last one shows the economic results expected and the generated profits. The article ends by pointing out the critical factors of the business plan: the characteristics of the demand in the destination market and the supply of raw material.
\end{abstract}

Key words: squid, breaded seafood, exportation, United States.

* Basado en la tesis del mismo título presentada por los autores a la Universidad ESAN en el 2006 para optar de grado de Magíster en Administración. 


\section{Introducción}

A pesar de la larga tradición pesquera del Perú y la riqueza de su mar, un rasgo característico de la industria pesquera nacional es su orientación a la producción y la exportación de materias primas.

En los últimos años, el calamar gigante, especie que destaca dentro de la variedad de recursos que ofrece el mar peruano por su abundancia y resistencia a fenómenos climáticos, ha desempeñado un papel protagónico en las exportaciones nacionales. Así, el país participó durante el 2004 con el 40\% de la oferta mundial del recurso.

No obstante la abundancia e importancia del recurso, sólo el 2\% de las exportaciones totales de calamar gigante se realiza bajo una presentación que implica valor agregado. El mayor porcentaje de las exportaciones se compone de materia prima y productos intermedios; es decir, se mantiene el mismo patrón de otros productos que forman parte de la oferta exportable nacional.

En ese contexto, el objetivo de este trabajo es desarrollar la oportunidad de negocio que se genera a partir del procesamiento local del recurso calamar gigante y de su colocación en el mercado estadounidense bajo la presentación de empanizados marinos. Como se demostrará más adelante, esta oportunidad de negocio no solo es rentable y factible desde los puntos de vista económico y de operaciones, sino que es propicia para la generación de valor $\mathrm{y}$ el ingreso en el mercado internacional de productos terminados. El beneficio es evidente, pues permite obtener un precio superior en $800 \%$ al que paga actualmente el mercado internacional por el recurso bajo la presentación de materia prima.

\section{Situación del recurso en el Perú}

El calamar gigante (Dosidicus gigas) es una de las diversas especies de calamarIllex, Todarodes pacificus, Ommaestrephes Bartrami, entre otras- que se comercializan en el mundo.

En el Perú, la extracción de la especie se inició en la zona norte, y recién a partir de la década del noventa empezó a cobrar importancia por la cantidad de biomasa disponible para su captura, realizada básicamente por la flota artesanal. Aun en la actualidad, los mayores desembarques se presentan en esa zona; sin embargo, la especie también se captura en la zona sur, en el puerto de Matarani.

En el año 2004, la extracción de calamar gigante alcanzó 250 mil toneladas, lo que significó un incremento de $96 \%$ con relación al año anterior. En promedio, en los últimos cinco años, la captura de la especie se ha incrementado en 117 mil toneladas (ver cuadro 1); más del 70\% proviene de la pesca artesanal.

A pesar de la abundancia de la especie, el consumo interno es muy bajo, pues más del $85 \%$ del total de los desembarques se destina al comercio exterior. Esto lo convierte en el principal producto de exportación de la pesca de consumo humano directo (CHD). Las exportaciones de calamar alcanzaron los 88 millones de dólares en el año 2004 (ver gráfico 1) y se espera que al término de 2005 asciendan a 108 millones de dólares, lo cual implicaría un crecimiento de $22 \%$ en los últimos cinco años.

Con relación al precio, este ha mantenido una tendencia constante en los últimos años, luego del descenso que se observó a partir del 2000. Se estima que para el 
Cuadro 1. Desembarques de calamar gigante

\begin{tabular}{|c|c|c|c|c|c|c|c|}
\hline & \multicolumn{6}{|c|}{ Histórico } & \multirow{2}{*}{$\begin{array}{r}\text { Crecimiento } \\
\text { promedio } \\
1999-2004\end{array}$} \\
\hline & 1999 & 2000 & 2001 & 2002 & 2003 & 2004 & \\
\hline Desembarco de calamar gigante (miles t) & 54,7 & 53,8 & 71,8 & 146,4 & 128,0 & 250,8 & 117,6 \\
\hline Variación \% & 9891,2 & $-1,6$ & 33,5 & 103,8 & $-12,6$ & 96 & 177,7 \\
\hline $\begin{array}{l}\text { Exportación de calamar gigante } \\
\text { (millones US\$) }\end{array}$ & 19,7 & 19,4 & 25,8 & 40,3 & 40,7 & 88,1 & 39 \\
\hline $\begin{array}{l}\text { Variación \% } \\
\text { Tradicionales pesqueras } \\
\text { Variación \% } \\
\text { No tradicionales pesqueras } \\
\text { Variación \% }\end{array}$ & $\begin{array}{r}6494,9 \\
600,9 \\
46,6 \\
189,6 \\
-15,7\end{array}$ & $\begin{array}{r}-1,8 \\
953,8 \\
58,7 \\
186,4 \\
-1,7\end{array}$ & $\begin{array}{r}33,1 \\
926,2 \\
-2,9 \\
208,2 \\
11,7\end{array}$ & $\begin{array}{r}56,5 \\
892,3 \\
-3,7 \\
163,7 \\
-21,4\end{array}$ & $\begin{array}{r}0,9 \\
821,0 \\
-8,0 \\
203,8 \\
24,5\end{array}$ & $\begin{array}{r}116,3 \\
1103,2 \\
34,4 \\
277,4 \\
36,1\end{array}$ & $\begin{array}{r}157,9 \\
882,9 \\
17,9 \\
204,8 \\
3,6\end{array}$ \\
\hline $\begin{array}{l}\text { Participación porcentual } \\
\text { (no tradicionales) }\end{array}$ & 10,4 & 10,4 & 12,4 & 24,6 & 20,0 & 36,4 & 18,3 \\
\hline
\end{tabular}

Fuente: Maximixe.

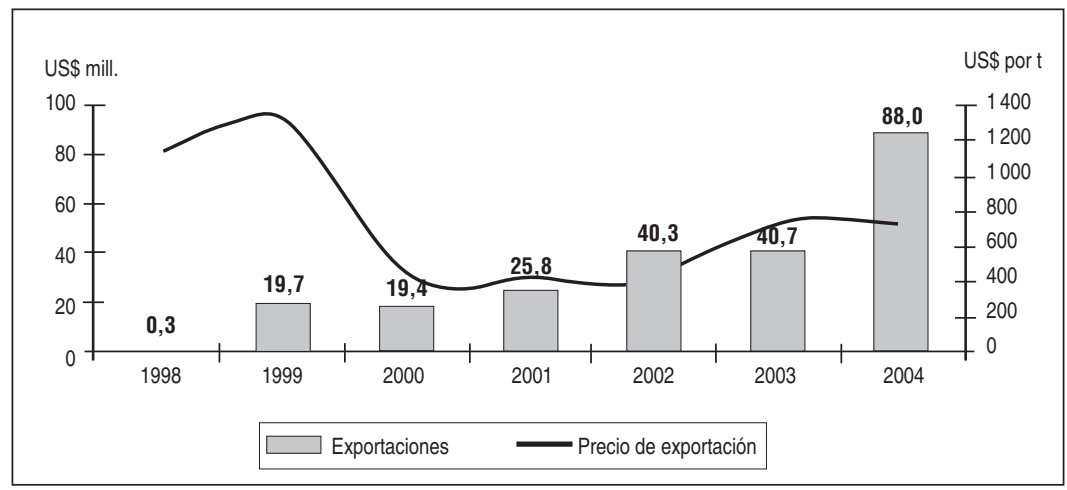

Gráfico 1

Exportaciones y precios del calamar gigante (1998-2004)

2005 el precio bordeará los 700 dólares por tonelada.

El calamar gigante se exporta en la modalidad de congelado en diversas presentaciones: filetes, alas, anillas, titas, hamburguesas, nuggets, entre otras. De los productos mencionados, los filetes crudo y precocido lideran las exportaciones con un total de 20 mil toneladas. Las presentaciones tipo anillas, aros, hamburguesas y nuggets han tenido aceptación en diversos mercados (ver gráfico 2), como España, Estados Unidos, Japón, Corea del Sur, entre otros, lo cual incentivó a diversos empresarios a incursionar en la elaboración de productos procesados de calamar gigante. Esta decisión ha permitido que en la actualidad se cuente con 30 tipos de presentaciones diferentes. 


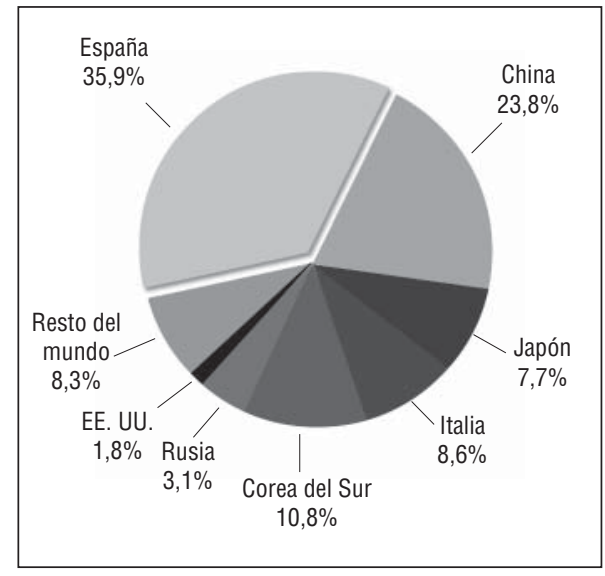

Fuente: Sunat.

\section{Gráfico 2}

Destino de las exportaciones de calamar congelado (2005)

Las empresas que elaboran productos empanizados de calamar u otra especie marina utilizan la tecnología existente en el Instituto Tecnológico Pesquero (ITP) para procesar sus productos. Esto se debe a que en el mercado peruano no hay ninguna empresa del sector pesquero que tenga una planta propia para realizar el procesamiento de empanizado de los productos.

\section{El modelo de negocio planteado}

El modelo de negocio que se propone abarca el aprovisionamiento de la materia prima en el puerto de Paita (por medio de los pescadores artesanales), la producción y el empaquetado en planta propia, y la exportación directa al mercado de California, Estados Unidos. El producto, empanizado marino de calamar gigante, será vendido a distribuidores mayoristas, quienes lo introducirán en el mercado con su propia marca o la del supermercado con el que operen.
El procesamiento local del recurso, previo a su exportación, posibilitará el acceso al mercado de productos terminados en Estados Unidos a un precio competitivo, lo que permitirá la generación de un margen de $47 \%$ como consecuencia del diferencial entre los costos locales y los precios pagados en el mercado objetivo.

El aprovisionamiento directo por medio de los pescadores artesanales permite tener control sobre la materia prima recién extraída y asegura que su estado sea fresco.

Según lo planeado, la planta será lo suficientemente flexible como para responder de manera rápida a las especificaciones del cliente, como la forma y el diseño del empanizado. Las formas comúnmente encontradas en la categoría de productos empanizados marinos en el mercado de destino son: nuggets, fingers, tenders, balls y pop corns. Todas estas, e incluso sus variantes, pueden fabricarse en la línea de producción contemplada, sin que se requiera inversión adicional.

\subsection{El producto: empanizados de calamar gigante}

Los empanizados de calamar gigante han sido concebidos de tal manera que puedan ingresar en el mercado objetivo con unas características y un precio que les permitan incorporarse a la variedad de empanizados marinos que actualmente se comercializan y se consumen.

La presentación es tipo nugget, listo para servir y consumir luego de descongelarlo brevemente y de calentarlo (en el horno de microondas o en el horno convencional) o freírlo. Los estándares establecidos por el mercado especifican que cada unidad tenga una forma rectangular y un peso promedio 
de 25 gramos. El producto se presenta en cajas de 300 y 600 gramos, lo que equivale a 12 y 24 unidades, respectivamente.

El consumidor final se beneficia no sólo de los atributos de practicidad, versatilidad en la preparación y oportunidad de consumo, sino también del valor nutricional del producto, debido a su contenido de vitaminas, minerales y aminoácidos. Además, el empaque -cajas de cartón doble parafinadas- facilita la manipulación y el almacenamiento del producto hasta por doce meses en refrigeración, lo cual permite conservar su calidad.

\subsection{El mercado de destino}

Estados Unidos, segundo importador mundial de productos marinos, constituye el mercado de destino. Según cifras de la National Marine Fisheries Service (NMFS), las importaciones registradas para el año 2004 alcanzaron un valor récord de 11,3 mil millones de dólares, cifra que superó a la del año anterior en 235,8 millones de dólares.

En cuanto a los empanizados marinos, el valor de las importaciones realizadas por Estados Unidos ascendió a 121 millones de dólares en el año 2004, lo cual representa un incremento de $48 \%$ en relación con el año 2003. En promedio, las importaciones de empanizados marinos han mantenido una tasa de crecimiento promedio anual de $43 \%$ en los últimos seis años.

Los empanizados de calamar gigante se comercializan en Estados Unidos en la categoría de productos congelados, segmento líder en ventas de todas las categorías de productos en dicho país. Según estudios realizados por la compañía norteamericana Mintel Internacional Group, la categoría de productos congelados marinos crecerá en aproximadamente $53 \%$ en el periodo 2004 2009, lo que significaría un incremento anual de $8 \%$ o $9 \%$. Dicho crecimiento se encuentra asociado a una mayor presencia de población hispana, segmento que muestra preferencia por los productos marinos.

En cuanto a la participación de los diferentes estados de ese país en las importaciones totales de productos elaborados a partir del calamar, el estado de California se ubica en el primer lugar: en el año 2004 absorbió el 55\% del total.

Por otro lado, desde el punto de vista demográfico y comercial, el estado de California presenta características (ingreso per cápita, composición poblacional, hábitos de consumo y tráfico comercial) que lo convierten en un mercado atractivo y de fácil acceso para la introducción del producto propuesto.

\subsection{Grupo de clientes}

Los distribuidores mayoristas de alimentos marinos que abastecen las cadenas de supermercados en Estados Unidos, en particular en el estado de California, serán los clientes objetivo a quienes se dirigirá la oferta exportable.

El producto está concebido para ser comercializado con la propia marca de los clientes. Esta estrategia demanda menores costos de márketing y logística, a la vez que aprovecha el conocimiento del mercado, las relaciones comerciales y las cadenas de distribución. Cabe señalar que los distribuidores mayoristas abastecen al $50 \%$ de los comerciantes minoristas en Estados Unidos, mientras que la venta directa del fabricante representa el $25 \%$. 


\subsection{Plan de márketing}

El planeamiento de la estrategia de márketing, según los alcances trazados para este plan de negocios, define como objetivo principal la introducción del producto en el mercado objetivo. A partir de ahí, se delinearon las estrategias del producto, la promoción, el precio, la distribución y las ventas, las que se presentan a continuación de manera resumida.

\section{Definición del producto}

Dentro de la amplia variedad de productos marinos congelados se ha escogido la presentación de empanizados como respuesta a la necesidad, cada vez mayor, del mercado de adquirir productos prácticos en su preparación y de fácil conservación, principalmente porque el número de mujeres que trabajan fuera de su casa es cada vez mayor y requieren productos nutritivos y de rápida preparación.

La selección de las presentaciones que se utilizarán como herramienta promocio- nal para los clientes objetivo se basó en un sondeo de productos realizado en Estados Unidos. Dicho sondeo consistió en la recopilación de información sobre las marcas y las presentaciones de empanizados marinos disponibles en el mercado, en los supermercados de los estados de California, Florida, Texas, Indianápolis y Washington.

Si se tiene en cuenta que la estrategia propuesta por el plan de negocios es la introducción de un producto estándar que resulte de rápida aceptación en el mercado objetivo, el diseño del producto obedecerá a los resultados del análisis de los datos recopilados en el mismo mercado objetivo mediante el sondeo de productos.

A continuación se presentan dichos resultados, que han sido clasificados en función de las siguientes características:

- Forma. Nuggets rectangulares de aproximadamente tres centímetros de largo por dos centímetros de ancho y un espesor promedio de un centímetro. El peso promedio es de 25 gramos y la cubierta

\section{Nuggets de pollo}

\begin{tabular}{|c|c|c|}
\hline \multicolumn{3}{|c|}{\begin{tabular}{|l} 
Nutrition Facts \\
Serving Size $28 \mathrm{~g}$
\end{tabular}} \\
\hline \multicolumn{3}{|l|}{ Amount Per Serving } \\
\hline \multirow[t]{2}{*}{ Calories 76} & Calories fron & i Fat 31 \\
\hline & $\%$ Daily & value* \\
\hline \multicolumn{2}{|l|}{ Total Fat $3 \mathrm{~g}$} & $5 \%$ \\
\hline \multirow{2}{*}{\multicolumn{3}{|c|}{ Saturated Fat $1 \mathrm{~g}$}} \\
\hline & & \\
\hline \multicolumn{2}{|l|}{ Cholesterol $31 \mathrm{mg}$} & $10 \%$ \\
\hline \multicolumn{3}{|l|}{ Sodium $163 \mathrm{mg}$} \\
\hline \multicolumn{3}{|c|}{ Total Carbohydrate 7} \\
\hline \multicolumn{3}{|l|}{ Dietary Fiber $0 \mathrm{~g}$} \\
\hline \multicolumn{3}{|l|}{ Sugars $1 \mathrm{~g}$} \\
\hline \multicolumn{3}{|l|}{ Protein $4 \mathrm{~g}$} \\
\hline Vitamin A $1 \%$ & Vitamin C & $0 \%$ \\
\hline Calcium $\quad 1 \%$ & Iron & $1 \%$ \\
\hline \multicolumn{3}{|c|}{$\begin{array}{l}\text { *Percent Daily Are based on a } 2,000 \text { calorie } \\
\text { diet.Your daily values may de higger or lower } \\
\text { depending on your calorie needs. }\end{array}$} \\
\hline \multicolumn{3}{|c|}{ NutritionData.com } \\
\hline
\end{tabular}

Fuente: http:// www.nutritiondata.com
Sticks de calamar

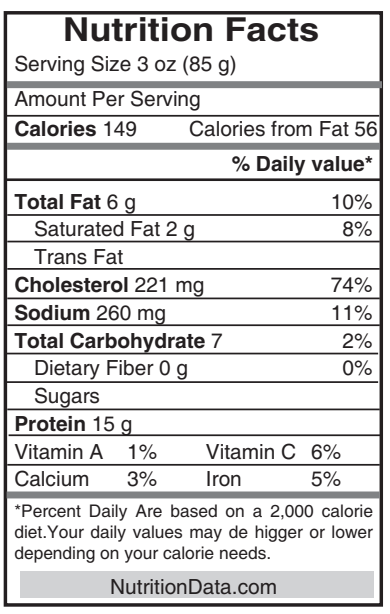

\section{Sticks de pescado}

\begin{tabular}{|c|c|c|c|}
\hline \multicolumn{4}{|c|}{$\begin{array}{l}\text { Nutrition Facts } \\
\text { Serving Size } 6 \text { pieces }(96 \mathrm{~g})\end{array}$} \\
\hline \multicolumn{4}{|c|}{ Amount Per Serving } \\
\hline \multirow{2}{*}{\multicolumn{2}{|c|}{ Calories 285}} & Calories from & Fat 163 \\
\hline & & \% Daily & value* \\
\hline \multicolumn{3}{|c|}{ Total Fat $18 \mathrm{~g}$} & $28 \%$ \\
\hline \multicolumn{3}{|c|}{ Saturated Fat $4 \mathrm{~g}$} & $19 \%$ \\
\hline \multicolumn{4}{|c|}{ Trans Fat } \\
\hline \multicolumn{3}{|c|}{ Cholesterol $53 \mathrm{mg}$} & $18 \%$ \\
\hline \multicolumn{3}{|c|}{ Sodium $551 \mathrm{mg}$} & $23 \%$ \\
\hline \multicolumn{3}{|c|}{ Total Carbohydrate 1} & $5 \%$ \\
\hline \multicolumn{3}{|c|}{ Dietary Fiber $1 \mathrm{~g}$} & $3 \%$ \\
\hline \multicolumn{4}{|c|}{ Sugars $1 \mathrm{~g}$} \\
\hline \multicolumn{4}{|c|}{ Protein $15 \mathrm{~g}$} \\
\hline \multicolumn{4}{|c|}{ Vitamin A $\quad 0 \%$} \\
\hline \multicolumn{4}{|c|}{$\begin{array}{llll}\text { Calcium } & 1 \% & \text { Iron } & 4 \%\end{array}$} \\
\hline \multicolumn{4}{|c|}{$\begin{array}{l}\text { *Percent Daily Are based on a } 2,000 \text { calorie } \\
\text { diet.Your daily values may de higger or lower } \\
\text { depending on your calorie needs. }\end{array}$} \\
\hline \multicolumn{4}{|c|}{ NutritionData.com } \\
\hline
\end{tabular}


está compuesta por empanizado de pan rallado o miga de pan procedente de una masa extrudida, horneada y seca.

- Color. La cubierta exterior será de color dorado, de textura fina y homogénea. El color interno será blanco, de apariencia homogénea y textura suave, según lo han confirmado los representantes de las empresas nacionales que actualmente exportan al mercado estadounidense.

- Sabor. Neutro, poco condimentado, manteniendo el sabor de los empanizados tradicionales, de manera que resulten atractivos para el público de diferentes edades. Esta característica no solo se deriva de los resultados obtenidos en el sondeo de productos, sino también de las experiencias de comercialización de empresarios locales. En el sondeo realizado se observó que el $72 \%$ de las presentaciones mantiene el empanizado tradicional, mientras que el $28 \%$ restante tiene algún tipo de condimentación.

- Empaque. Las presentaciones que actualmente se encuentran en el mercado estadounidense y que son producidas por empresas locales comprenden dos tipos de empaque: bolsas y cajas para refrigeración. Esta última constituye la presentación más común. Sobre dicha base se definió que las muestras se confeccionarán con cajas de cartón doble, parafinado, con un arte final multicolor, de acuerdo con el diseño seleccionado.

- Calidad. El producto final tendrá una calidad de grado A, según los estándares de la Food and Drugs Administration (FDA).

- Presentación. La selección de la presentación partió del análisis de los datos obtenidos en el sondeo de productos, donde se identificaron dos oportuni- dades de introducción de los empanizados en el mercado: cajas de 300 y 600 gramos.

\section{Segmentación del mercado objetivo}

Como se señaló anteriormente, los clientes primarios serán distribuidores que comercializan en el mercado de Estados Unidos con marca propia o de los supermercados. Estos clientes pueden segmentarse en función de diferentes criterios: la cartera de productos, la especialización, el tipo de especie que comercializan, la zona de cobertura, la forma como comercializan sus productos y la procedencia de estos.

Para fines de este plan de negocios, la segmentación se realizó de acuerdo con la especialización (productos marinos), la forma como comercializan los productos (procesados) y la zona de cobertura (estado de California).

Sobre la base de los criterios anteriores se llegó a un listado de 61 posibles clientes, entre los cuales se encuentran cinco cadenas de supermercados que actúan como sus propios distribuidores con productos de marca propias; los dos principales distribuidores de alimentos en Estados Unidos, Sysco y US Food Service; y los tres principales distribuidores nacionales de comidas congeladas: Supervalu Inc., Fleming Co y C\&S Wholesale Grocers.

A pesar de que el alcance de este plan de negocios no llega hasta la colocación directa del producto en el punto de venta minorista ni, por ende, al consumidor final, es importante su estudio porque este último impulsará o no la demanda, dependiendo del grado de aceptación que se logre. Por este motivo, se ha considerado como clientes secundarios los segmentos de amas de casa, 
niños, adultos jóvenes, adultos mayores y mujeres que trabajan.

Los grupos más relevantes son los adultos jóvenes y las mujeres que trabajan. La importancia del primer grupo reside en que por su rango de edad (25 y 35 años) y las tendencias que ha seguido el mercado norteamericano desde los años setenta, estos individuos se encuentran familiarizados con los empanizados desde su infancia y ahora los incluyen en su dieta.

El segundo grupo, mujeres que trabajan, se ha incrementado en número en Estados Unidos; así, por ejemplo, en el año 2003, el 53,7\% de las madres con hijos formaban parte de la población económicamente activa y el $56,2 \%$ de las madres solteras también desempeñaba trabajos fuera del hogar. Además, estudios sobre los hábitos de consumos de la población estadounidense reconocen la importancia del papel que desempeña la mujer al momento de realizar las compras en los supermercados, razón por la cual es fundamental que en el plan de márketing desarrollado se incluya un seguimiento a las necesidades de este grupo.

\section{Estrategia de precios}

La estrategia de precios parte del sondeo de productos realizado en Estados Unidos y sigue la estrategia general del plan de negocios de introducir un producto estándar en relación con la oferta existente.

Al analizar los datos obtenidos en el sondeo de productos, se observó que se encontraban distribuidos en torno a dos presentaciones: las de menor tamaño, con un peso de alrededor de 300 gramos, y las más grandes, con un peso aproximado de 600 gramos. En el análisis realizado en conjunto, la variable precio no mostró correlación con el peso, razón por la cual se trabajó de manera individual. De esta manera se logró identificar dos oportunidades de penetración: cajas de 300 y 600 gramos, a precios promedio de mercado de 4,8 dólares y 5,6 dólares, respectivamente.

El precio de fábrica se determinó en función del precio estimado de venta en los supermercados, mediante el uso del método de Cost-plus escalation. En el caso de la presentación de 300 gramos, se estableció que el plan es rentable con un precio de fábrica de 2,76 dólares, si la venta se realiza al mayorista, lo cual implicaría que la venta directa al supermercado tendría un resultado aun más atractivo. Sin embargo, dicha opción no ha sido evaluada en este plan de negocios porque la penetración por medio de ese canal requiere un mayor conocimiento del mercado en comparación con el que se tiene en la etapa de introducción del producto. En el caso de la presentación de 600 gramos, se determinó un precio de fábrica de 3,21 dólares, para un precio estimado de venta al minorista de 5,60 dólares.

\section{Distribución}

La distribución será de tipo intensiva, pues involucra a varios intermediarios, y tendrá como cliente a los mayoristas de alimentos en el mercado de Estados Unidos, quienes se encargarán de vender el producto a los minoristas. Reconociendo el hecho de que la empresa no puede competir con los volúmenes de producción de las empresas líderes, una alternativa atractiva sería la comercialización por medio de los distribuidores de las cadenas de supermercados más pequeñas (Wild Oats, Whole Foods yTrader Joes, entre otras), con una oferta de productos distintiva. Cabe señalar que dicho segmento no es atractivo para los líderes del mercado. 


\section{Promoción y publicidad}

La estrategia de promoción y publicidad contempla la programación de actividades de comunicación y acceso a los potenciales clientes.

En un inicio, los medios de comunicación por utilizar serán los siguientes: carta de presentación, catálogo de productos o folletos de propaganda (brochures), página web, muestras, degustaciones y participación en ferias. Estas últimas son particularmente importantes para la penetración en dicho mercado, pues en ellas se reúnen todas las personas del sector-desde productores hasta comercializadores y empresas relacionadas y de soporte-, lo que proporciona una oportunidad única de tener acceso a una gran cantidad de contactos. En ese sentido, las principales ferias estadounidenses a las que se asistirá son: International West Coast Seafood Show (WCSS), California e International Boston Seafood Show.

\subsection{Estrategia de operaciones}

El diseño de las operaciones se orienta a identificar los procesos de producción, logísticos y de servicios más adecuados para la elaboración de los empanizados de calamar gigante con las características determinadas en el plan de márketing. La estrategia de operaciones abarca dos etapas: una primera preoperativa, en la cual se definen las variables más relevantes que condicionarán el futuro desempeño del proyecto, en función de los objetivos planteados; y una segunda operativa, que contempla las actividades primarias de la cadena de valor, desde el acopio de la materia prima hasta la logística de salida.

\section{Etapa preoperativa}

A continuación se presentará, de manera resumida, las variables consideradas en esta etapa, ordenadas según su importancia relativa.

- La infraestructura del procesamiento. $\mathrm{Su}$ determinación implicó establecer contacto con la empresa Tecnofood S.A.C., representante de la marca Convenience Food System, por ser el proveedor más importante de tecnologías de empanizado en el país. Se optó por la línea de menor capacidad, con la finalidad de reducir los requerimientos de inversión inicial y, por tanto, el riesgo del negocio. Esta línea produce a un ritmo de 290 kilogramos por hora y define la máxima capacidad de la planta en 142 toneladas mensuales de producto terminado.

Un aspecto importante de la tecnología de procesamiento es la flexibilidad que tiene para adaptar las características del producto terminado a los requerimientos específicos de los clientes. Estas exigencias pueden variar en cuanto a la forma -que está definida por un moldey a la formulación-que está en función de la composición de los insumos- de los empanizados.

La línea de procesamiento se complementa con la instalación de dos cámaras de refrigeración para el almacenamiento de la materia prima y los productos terminados. Ambas tendrán una capacidad de almacenamiento de 30 toneladas, operarán a $-25^{\circ} \mathrm{C}$ y con una humedad de $73 \%$, de conformidad con los requerimientos del Departamento de Comercio de Estados Unidos. 
- La localización de la planta. En este caso se seleccionaron como factores determinantes: las características del recurso, la continuidad en el abastecimiento del recurso, la frecuencia de arribos y partidas de los buques de transporte, el acceso a medios de transporte y comunicación, la cercanía a los proveedores de otros insumos y, finalmente, el riesgo social o político de la zona. La evaluación de las posibles localizaciones se realizó sobre la base de las visitas de campo y la opinión de expertos. Luego de realizar una ponderación de los factores de localización, se obtuvo como resultado que los puertos de Paita, Talara y Matarani son los más importantes para la extracción del calamar gigante (véase cuadro 2).

Finalmente, la localización seleccionada para el acopio de la materia prima y la instalación de la planta de procesamiento es el puerto de Paita, porque registra el mayor volumen de extracción artesanal del calamar gigante en el país $(56 \%)$, con una oferta diaria promedio de 370 toneladas, y porque presenta, en su zona industrial, la más importante concentración de plantas de procesamiento para este recurso. Además, esta concentración ha impulsado el desarrollo de industrias complementarias, como la producción de hielo y los servicios de transporte refrigerado, que incrementan el atractivo de Paita como centro de operación.

- El inicio de una estrategia de acercamiento con los pescadores artesanales. Puesto que son los principales proveedores del recurso calamar gigante, la finalidad es establecer con ellos relaciones de largo plazo que aseguren el abastecimiento continuo de la materia prima. De las visitas de campo realizadas a los puertos de Paita y Talara, se pudo concluir que sus principales requerimientos son los siguientes:

- Un precio competitivo, para evitar la eventual migración hacia la extracción de otros recursos de mayor valor comercial. Sobre este punto es importante señalar que los resultados económicos proyectados del plan de negocios permiten incrementos en el costo de la materia prima de hasta veinte veces el costo actual, manteniendo un valor actual neto positivo. Cabe señalar que la viabilidad económica del proyecto es insensible a las variaciones del precio de la materia prima.

- Relaciones de largo plazo, con el fin de obtener una mayor estabilidad en sus labores de extracción, lo

\section{Cuadro 2. Matriz de localización}

\begin{tabular}{lccccccc}
\hline Variable & Peso & \multicolumn{2}{c}{ Talara } & \multicolumn{2}{c}{ Paita } & \multicolumn{2}{c}{ Matarani } \\
\hline Continuidad en el abastecimiento & $35 \%$ & 3 & 1,05 & 3 & 1,05 & 1 & 0,35 \\
Frecuencia de buques internacionales & $30 \%$ & 0 & 0,00 & 3 & 0,90 & 3 & 0,90 \\
Tamaño del recurso & $15 \%$ & 2 & 0,30 & 2 & 0,30 & 3 & 0,45 \\
Acceso a medios de transporte y otros servicios & $15 \%$ & 1 & 0,15 & 3 & 0,45 & 3 & 0,45 \\
Riesgos sociales/políticos & $5 \%$ & 3 & 0,15 & 2 & 0,10 & 2 & 0,10 \\
\hline & $100 \%$ & \multicolumn{2}{c}{1,65} & & $\mathbf{2 , 8 0}$ & & 2,25 \\
\hline
\end{tabular}

Elaboración propia. 
cual permite cumplir con los requerimientos diarios de materia prima.

- Aprovisionamiento diario de hielo para sus embarcaciones, el cual ha sido considerado en los presupuestos y la evaluación económica.

\section{Etapa operativa}

- Logística de entrada. El procesamiento de un día de empanizados, utilizando el máximo de la capacidad instalada de la planta, necesita 6,8 toneladas de materia prima, menos del $2 \%$ de la oferta artesanal diaria en el puerto de Paita, lo cual reduce el riesgo de desabastecimiento. Además, se cuenta con la ventaja que la extracción se realiza durante todo el año, pues no existe periodo de veda para este recurso.

El calamar se limpia y se corta en el mismo puerto. La comercialización se realiza bajo tres presentaciones y precio, según el corte: manto (o tubo), aleta y tentáculo (o rejo). Tanto el tentáculo como la aleta distorsionan las propiedades del producto terminado, pues el tentáculo presenta una coloración rojiza que se mantiene hasta el producto final y la aleta tiene una contextura más gruesa. Frente a esta situación, se ha consi- derado comprar únicamente el manto, porque reúne las mejores características organolépticas para la elaboración del producto final.

- Operaciones. Esta actividad es relevante en la generación de valor del proyecto porque incorpora la transformación del calamar gigante en la presentación final, aquella con la cual será introducido en el mercado objetivo.

La elaboración se inicia en la planta de procesamiento, con la recepción de la materia prima, y continúa con las operaciones de limpieza, pesado, cortado y laminado.

Las operaciones en la línea de procesamiento se inician con el descongelado de los bloques de manto, seguido del desmenuzado y mezclado con una serie de ingredientes. Este procedimiento permite obtener una masa fría, que será moldeada según el formato requerido. Las porciones resultantes se someten a una operación de rebozado para permitir la adherencia de las migas de pan, que serán aplicadas en el siguiente proceso. Las porciones empanadas se someten a una fase previa de freído. Después de un breve periodo de enfriamiento, el producto se somete a un proceso de conge-

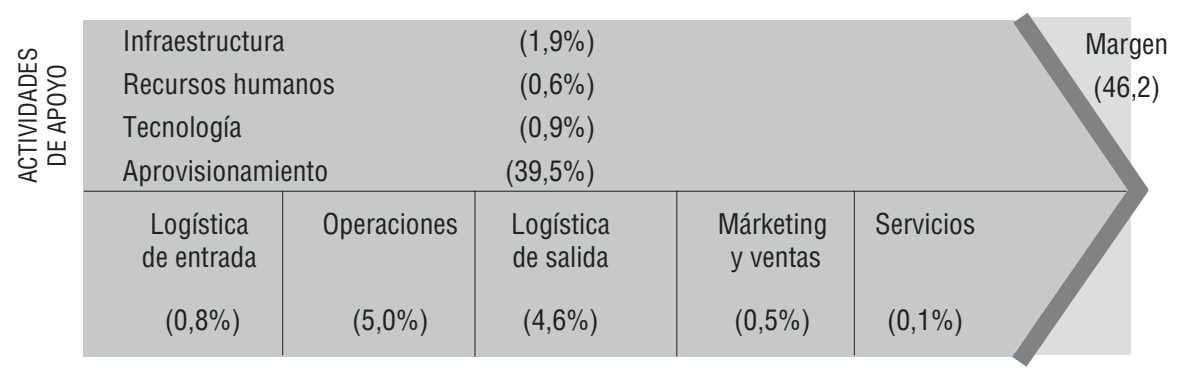

ACTIVIDADES PRIMARIAS

Gráfico3

Cadena de valor 
lación continua $\left(\mathrm{a}-30^{\circ} \mathrm{C}\right)$. Por último, el producto es empaquetado en cajas de cartón doble parafinadas.

El producto en su presentación final se conserva en cámaras de almacenamiento de productos congelados, a $-25^{\circ} \mathrm{C}$, hasta acumular las 19 toneladas necesarias para llenar un contenedor e iniciar las operaciones de logística de salida.

- Logística de salida. Esta etapa se inicia con la reserva del espacio o booking, en la naviera seleccionada para la ruta de destino (Paita-Los Ángeles) en un contenedor reefer de cuarenta pies. Los contenedores preenfriados son enviados a la planta, donde se procede al estibado de la mercadería y se coloca el precinto de seguridad. Luego se traslada la mercadería en el contenedor reefer, que mantiene la carga entre temperaturas de $-18{ }^{\circ} \mathrm{C}$ y $22^{\circ} \mathrm{C}$. En ese momento se realiza la descarga de la mercadería, del contenedor al buque.

El tiempo de travesía del puerto de Paita al de Los Ángeles es de diez días aproximadamente, y la frecuencia de buques de carga es semanal. El plan de negocios contempla la tercerización de las actividades de logística de salida, pues estas pueden ser realizadas por un operador logístico de primer nivel.

- Comercialización. Las condiciones de venta consideran la extensión de una carta de crédito a la vista e irrevocable contra la presentación de documentos por el $60 \%$ del contrato, y el $40 \%$ restante contra la aprobación de la FDA. Cost and Freight o Costo y Flete (CFR) es el Incoterm más utilizado en la exportación de este tipo de productos a Estados Unidos, pues los distribuidores consiguen mejores condiciones en el seguro mediante el aprovechamiento de las economías de escala de sus volúmenes de importación.

\subsection{Evaluación económica}

La propuesta del plan de negocio en cuanto a producto, mercado y factibilidad técnica ya ha sido desarrollada. A continuación, se muestran los resultados económicos esperados y la rentabilidad generada.

\section{Inversión requerida}

La evaluación económica se ha realizado tomando en cuenta un horizonte de 10 años y con la aplicación de perpetuidades utilizando la metodología de valor de desecho.

Las inversiones ascienden a 1,4 millones de dólares y se efectúan en el periodo preoperativo y en el primer año. La mayor parte, el $75 \%$, se destina a activos fijos, entre los cuales sobresalen la línea de procesamiento y los demás equipamientos para la planta respectiva.

La inversión en capital de trabajo ha sido calculada sobre la base de los saldos negativos del primer periodo y de las necesidades del inicio de la actividad productiva. Es importante recalcar que el plan contempla que las actividades de promoción y búsqueda de clientes se inicien en el periodo preoperativo y abarquen todo el primer año.

Las inversiones en intangibles están relacionadas con la consecución de las licencias y las certificaciones necesarias para el inicio de las operaciones de producción y el ingreso del producto en el mercado estadounidense. 


\section{Proyección de ingresos y egresos}

El flujo de ingresos está compuesto por las ventas. En vista de que todo el primer año y parte del periodo preoperativo se destinará a la búsqueda de clientes, se prevé que las ventas se iniciarán recién en el segundo año.

La proyección de las ventas se realizó bajo el supuesto de un escenario conservador, lo que dio como resultado un nivel de ventas inicial de seis contenedores anuales o medio contenedor mensual, lo que equivale al mínimo lote que puede ofertarse al mercado exterior. Es necesario señalar que una orden promedio de esta categoría de productos de un cliente mediano es de aproximadamente quince toneladas anuales.

Por otro lado, este nivel de ventas representaría apenas el $0,09 \%$ del total de importaciones de empanizados marinos del mercado objetivo. Se estima que el comportamiento de las ventas mantendrá una relación creciente, en función de los esfuerzos y constantes inversiones en márketing y los efectos del crecimiento del mercado.

Continuando con el escenario conservador, se calcula que las ventas se estabilizarán a partir del sexto año, en 42 contenedores anuales, con lo cual se habrá alcanzado una participación del $0,18 \%$ en las importaciones del mercado objetivo.

Los flujos de egresos se componen de costos y gastos. Con relación a los costos, los conceptos de empaques y embalajes son los que tienen mayor participación en el total y están incluidos en los costos indirectos de fabricación. Es importante señalar que si bien la materia prima representa el $77 \%$ de la composición del producto terminado, en términos de su costo, significa solo el $1 \%$ del precio de venta. Respecto de los gastos, los rubros correspondientes a márketing y ventas son los de mayor importancia. Según el presupuesto diseñado, estos comprenden: la participación en ferias, los viajes de visita a los posibles clientes, el envío de muestras y las diversas maneras de promoción que permitan lograr una cartera de clientes acorde con el nivel de ventas esperado.

Para tener una idea más concreta de la participación de los costos y gastos en el valor del producto terminado, se puede decir que ambos rubros representan el $43 \%$ de este, con lo que el margen operativo sería de $57 \%$. Este es el margen que resulta no solo del procesamiento de la materia prima, sino de la agregación de valor y su colocación en el mercado internacional como producto terminado.

La evaluación de la rentabilidad ha considerado una tasa de descuento del $17 \%$. Esta permite obtener un valor económico neto esperado ascendente a 5,3 millones de dólares, así como generar una tasa interna de retorno del $48 \%$. Los flujos son positivos a partir del segundo año y la recuperación de la inversión se inicia al finalizar el cuarto año. Además, se han identificado las variables con mayor impacto en los resultados económicos del plan: la variación del precio, los márgenes de los canales de distribución y el nivel de respuesta del mercado objetivo. En este sentido, la evaluación económica del proyecto incorporó el análisis de escenarios que simulen niveles de respuesta de mercado mínimos, así como variaciones en el precio y los márgenes, y se obtuvo resultados favorables.

Estos resultados demuestran que la factibilidad técnica del plan de negocios se complementa con una evidente rentabilidad y liquidez. 


\subsection{Factores críticos}

Los factores que pondrían en riesgo el alcance de los resultados señalados se han agrupado en dos grandes aspectos: el riesgo relacionado con el mercado y el riesgo asociado a la continuidad de la oferta exportable, es decir, a la disponibilidad permanente de la materia prima.

Con relación al riesgo del mercado, este puede reducirse por el hecho de que el producto final está diseñado con el fin de que constituya un integrante más de la variedad de productos empanizados que se comercializan y se consumen en el mercado objetivo. Además, las características estándar del producto disminuirían el riesgo en la etapa de introducción. Adicionalmente, se ha previsto continuar con una observación constante del mercado, de tal manera que la flexibilidad que caracteriza al proceso acondicione la oferta a las nuevas características demandadas por el cliente. Así, se responderá con rapidez a cualquier nuevo requerimiento del mercado.
Por el lado del aprovisionamiento, este riesgo se atenuaría por la resistencia de la especie calamar gigante a los factores climáticos moderados y por la continuidad de su extracción. No obstante, el plan desarrollado resalta la importancia de realizar acuerdos o alianzas estratégicas con los pescadores artesanales, principales proveedores del recurso. La capacidad de realizar alianzas se refuerza con el margen generado por la diferencia entre los costos de la materia prima y el precio del producto terminado, lo cual permite ofrecer mayores precios a los pescadores $\mathrm{y}$, por ende, mejorar la posición en la relación establecida.

Los factores mencionados junto con el control de calidad y la coordinación logística constante que facilite la continuidad de la oferta exportable constituyen los factores críticos de éxito. En este caso, al igual que en todo negocio, las competencias del recurso humano son el factor determinante porque la calidad de la gestión es la que, en última instancia, posibilita que los planes se conviertan en realidad. 


\section{Bibliografía básica}

AMERICAN INSTITUTE OF FOOD DISTRIBUTORS (Instituto Americano de Distribuidores de Alimentos). 2005. Food distributors by region. <http://www.foodinstitute.com> (5 de nov. de 2005).

FOOD MARKETING INSTITUTE. (Instituto de Mercadeo de Alimentos). 2005. Consumer trend. $<$ http://www.fmi.org $>$ (5 de nov. de 2005).

GLOBEFISH. 2005. Commodity update: Cephalopods. <http://www.globefish.org> (15 de ago. de 2005).

INSTITUTO TECNOLÓGICO PESQUERO. 2004. Diagnóstico de la industria de transformación pesquera. Estudio de prospectiva para la cadena productiva de la industria pesquera en la región de la costa del Pacífico de América del Sur.

NATIONAL FISHERIES INSTITUTE (Instituto Nacional de Pesquería). 2005. Fishery products, per capita consumption. $<$ http://www. nfi.org $>$ (24 de nov. de 2005).

NORIEGA, Rocío. 2004. Tendencia del consumo de productos de mar en EE. UU. Amcham Chile.

PERÚ. COMISIÓN PARA LA PROMOCIÓN DE EXPORTACIONES. 2004. Estudio de mercado para productos a partir de la pota y especies similares. Lima: Prómpex. 